Musées, Patrimoine et Culture scientifiques et techniques

$176 \mid 2018$

mars-avril 2018

Entre santé publique et éthique patrimoniale : le poison dans les collections

\title{
Blandine Marcé
}

\section{OpenEdition \\ Journals}

Édition électronique

URL : http://journals.openedition.org/ocim/2033

DOI : 10.4000/ocim.2033

ISSN : 2108-646X

Éditeur

OCIM

Édition imprimée

Date de publication : 1 mars 2018

Pagination : 17-25

ISSN : 0994-1908

Référence électronique

Blandine Marcé, «Entre santé publique et éthique patrimoniale : le poison dans les collections », La

Lettre de I'OCIM [En ligne], 176 | 2018, mis en ligne le 01 mars 2019, consulté le 10 octobre 2020. URL http://journals.openedition.org/ocim/2033 ; DOI : https://doi.org/10.4000/ocim.2033

Ce document a été généré automatiquement le 10 octobre 2020

Tous droits réservés 


\title{
Entre santé publique et éthique patrimoniale : le poison dans les collections
}

\author{
Blandine Marcé
}

Four électrique cylindrique du dilatomètre différentiel enregistreur de Pierre Chenevard présentant des fibres d'amiante ( $n^{\circ}$ d'inventaire 20714-0001).

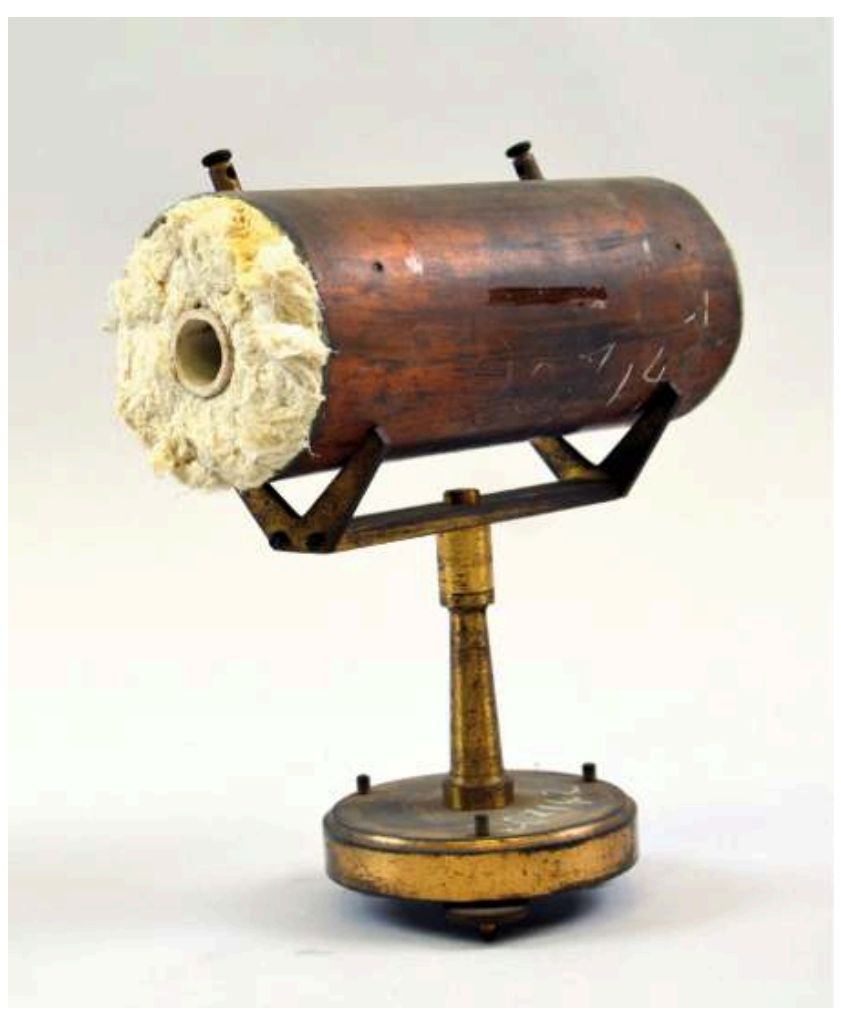

(C) Musée des Arts et Métiers, Cnam/Denis Pruvrel 
1 Les "collections poison", c'est-à-dire des "collections d'objets qui, de par les matériaux qui les constituent, ou les méthodes de conservation, de restauration et de préservation qui leur sont appliquées, présentent un danger pour la santé des professionnels et du public" suscitent, depuis une dizaine d'années, un intérêt croissant dans les publications scientifiques.

2 Les biens culturels contenant des traces d'arsenic, de plomb, de mercure, d'amiante, conservés dans du formol et radioactifs sont en effet au cœur d'un débat mettant en balance les intérêts patrimoniaux des collections et des questions de santé publique. Entre exagération et minimisation des impacts, la méconnaissance de ces biens menace tout à la fois l'intégrité des œuvres concernées et la santé des professionnels du patrimoine, au rang desquels on ne compte pas seulement les conservateurs et les restaurateurs, mais également les équipes de ménage, les régisseurs et le public. Entre éthique patrimoniale et questions de santé publique, quelle gestion proposer pour les "collections poison"?

\section{La toxicité}

La toxicité est la capacité d'une substance à produire des effets néfastes sur la santé. Dans le cadre des "collections poison", on parle plus souvent de toxicité chronique, correspondant à un empoisonnement causé par l'accumulation de petites doses de poison dans l'organisme sur plu---

sieurs mois et années, que de toxicité aiguë, où l'empoisonnement est réalisé à court terme, du fait d'une dose létale. En France, la Valeur Moyenne d'Exposition (VME), détermine la concentration maximale admissible d'un produit dangereux dans l'air, avant que son effet soit nocif..

3 Réalisée dans le cadre d'un mémoire de recherche, cette étude s'appuie sur le témoignage de dix-huit professionnels soucieux d'encourager les mesures préventives et de sensibiliser sur le sujet des poisons. Établissements référents, le Muséum national d'Histoire naturelle, en la personne de Marc Herbin, et le musée des Arts et Métiers, représenté dans cette étude par Sylvie Maillard, du service inventaire, ont permis l'utilisation des photographies de leurs réserves et apporté leur assistance dans la réalisation de cet état des lieux des pratiques actuelles, ayant pour objectif d'offrir des solutions pratiques à court, moyen, et long terme.

\section{Les poisons dans les collections}

On parle de poison pour "toute substance vitale qui, introduite dans l'organisme à dose suffisante, détruit ou altère les fonctions vitales" (Larousse, 2017). De fait, la présence de poison dans les collections ne se limite pas aux objets volontairement empoisonnés du fait de leur usage (comme les flèches au curare), mais à tout objet qui contiendrait une substance toxique à une certaine dose. Pour créer cette distinction, les professionnels les désignent souvent sous le terme de "polluants" ou "biocides".

5 Cette définition élargit le spectre qu'englobe la problématique de la toxicité des collections. Bien des poisons existent dans les musées, et certains sont mieux identifiés que d'autres. L'arsenic est, par exemple, le poison le plus documenté : 63,75\% des professionnels interrogés l'ont cité dans le questionnaire ayant servi à élaborer cette 
étude. Appliqué sous forme de pâte à l'intérieur des peaux tannées dès le milieu du XVIII ${ }^{e}$ siècle, l'arsenic se retrouve actuellement à migrer à la surface de ces dernières et à se mêler à la poussière des spécimens non-dépoussiérés, comme l'a montré l'étude de Marie de Beaulieu sur le dépoussiérage d'un lémurien naturalisé ${ }^{\text {. }}$.

En seconde position vient le formaldéhyde, $50 \%$ des professionnels l'ont identifié comme un poison. Plus connu sous le terme de "formol" quand il est mélangé à de l'eau, de l'alcool et du camphre ou du phénol, le formaldéhyde est utilisé dans les collections anatomiques et zoologiques pour conserver les spécimens dits "en fluide". On le retrouve par ailleurs dans l'art contemporain chez des artistes comme Damien Hirst, pour conserver des œuvres telles que The Golden Calf (2008).

$743,5 \%$ des professionnels ont ensuite désigné le mercure, connu sous deux manifestations patrimoniales principales : dans les herbiers et dans les instruments de mesure. Le "sublimé corrosif", contenant du chlorure mercurique, a, par le passé, été badigeonné sur les herbiers et l'intérieur des peaux tannées pour assurer leur conservation. Grâce à son grand coefficient de dilatation thermique, le mercure liquide a été employé dans les thermomètres, baromètres et sphygmomanomètres (permettant de mesurer la tension artérielle). Très conductible, on en retrouve également dans les piles ou les ampoules à mercure.

8 Le plomb concerne $37,5 \%$ des réponses, et recouvre des champs très différents. Il est présent dans les collections industrielles, dans des batteries, les radiateurs, ou les poids de métiers à tisser, mais également dans les collections archéologiques, avec des objets comme les monnaies ou les cercueils en plomb, à l'image de celui de Louise de Quengo étudié à Toulouse pour l'Inrap entre 2011 et $2013^{2}$. On le retrouve, dans les collections de Beaux-Arts, dans les baies de plomb des vitraux, mais aussi dans des pièces d'art contemporain, à l'instar des œuvres d'Anselm Kiefer.

Tableau récapitulatif des Valeurs Moyennes d'Exposition (VME) et de la toxicité des poisons les plus courants dans les collections patrimoniales

\begin{tabular}{|c|c|c|c|c|}
\hline & Etat originel & $\begin{array}{l}\text { Dangerosite } \\
\text { ef méthode de contamination }\end{array}$ & Impacts & Toxicité \\
\hline Amiante & $\begin{array}{l}\text { Minéral cristallin } \\
\text { ó lexture fibreuse } \\
\text { (ehrysotile) }\end{array}$ & $\begin{array}{l}\text { Fibres invisiblos très volatiles } \\
\text { obsorbées par inhalation }\end{array}$ & $\begin{array}{l}\text { Abestose, ploques pleuroles, fibroses de } \\
\text { la plèvre, cancer broncho-pulmonaire, } \\
\text { mósothetiome pleural }\end{array}$ & $\begin{array}{l}\text { VME }=0,1 \text { fibre por } \mathrm{cm}^{2} \text { sur } \\
\text { une porriodo d'une houre }\end{array}$ \\
\hline $\begin{array}{l}\text { Arsonic (As) } \\
\text { Antydride orsénieux ou } \\
\text { trioxydo d'arsenic }\left(\mathrm{As}_{2}, \mathrm{O}, \mathrm{s}\right)\end{array}$ & $\begin{array}{l}\text { Cristoux de } \\
\text { covlour griso }\end{array}$ & $\begin{array}{l}\text { Poussierres } \\
\text { Inhalation, ingestion }\end{array}$ & $\begin{array}{l}\text { Itritason des yeux, de la peau ef des } \\
\text { voies rospirabites, impacto lo systome } \\
\text { nerveux, le fole et le système vasculaire }\end{array}$ & $\begin{array}{l}\text { CMR classo Cl } \\
\text { T+ } \\
\text { VME }=200 \mu \mathrm{m} / \mathrm{m}^{3} \text { d'air } \\
\text { pout lo trioxydo d'arsenic }\end{array}$ \\
\hline $\begin{array}{l}\text { Formaldéhyde } \\
\text { ou formol }\left(\mathrm{CH}_{2} \mathrm{O}\right)\end{array}$ & $\begin{array}{l}\text { Liquide dillué, prósent } \\
\text { entro } 4 \% \text { [solution do } \\
\text { conservation) et } 37 \% \\
\text { [solution do fixation] }\end{array}$ & $\begin{array}{l}\text { Dógagement do vopeurs toxiques } \\
\text { incolores } \\
\text { Absorption par inhalation ou par } \\
\text { voio culonbo }\end{array}$ & $\begin{array}{l}\text { Itritason des yeux et des voles } \\
\text { respiratoites, brovlures, cópholbes }\end{array}$ & $\begin{array}{l}\text { Cancérogène groupe } 1 \text { (CIRC) } \\
\text { Cancérogène groupe } 3 \\
\text { (normes européennes) } \\
\text { VME = 0,5 ppm ; } 00,0,61 \mathrm{mg} / \mathrm{m}^{2}\end{array}$ \\
\hline $\begin{array}{l}\text { Mercure }(\mathrm{Hg}) \\
\text { Chlorure mercurique }\left(\mathrm{HgCl}_{2}\right)\end{array}$ & $\begin{array}{l}\text { Forme liquide de } \\
\text { couleur orgentée à } \\
\text { tompérature ambionto }\end{array}$ & $\begin{array}{l}\text { Inhalafion de vopeurs de mercure } \\
\text { inodoces et incolores } \\
\text { Contamination par vole cutanbe }\end{array}$ & $\begin{array}{l}\text { Troubles du système norveux, des } \\
\text { reins, irritations culanées et des voles } \\
\text { respirctoires }\end{array}$ & $\begin{array}{l}\text { CMR groupe } 3 \\
\text { Reprotoxique et perturboteur } \\
\text { endoctinion } \\
\text { VME }=0,02 \mathrm{mg} / \mathrm{m}^{3}\end{array}$ \\
\hline Plomb (Pb) & Mótal & $\begin{array}{l}\text { Poussière de plamb dégrodé } \\
\text { Absorption par voies pulmonaires } \\
\text { ef digestives }\end{array}$ & Troubles digestifs, nevrologiques & $\begin{array}{l}\text { CMR calógorie } 1 \\
\text { VME }=0,10 \mathrm{mg} / \mathrm{m}^{2}\end{array}$ \\
\hline Rodioadivite & $\begin{array}{l}\text { Minéraux rodioactif's } \\
\text { comme l'uronium } \\
\text { et lo rodium }\end{array}$ & $\begin{array}{l}\text { Désintégration ou altération des } \\
\text { cellules por expositon prolongée } \\
\text { Ingestion ou inhalation de } \\
\text { poussières irradiées }\end{array}$ & Brôlures, vomissements, concer & $\begin{array}{l}\text { Premiers effets notables } \\
\text { à } 100 \mathrm{mSv} \text { (millisievert)/an. }\end{array}$ \\
\hline
\end{tabular}

(c) Blandine Marcé

9 Également à cheval entre différentes collections, les objets radioactifs font la part belle aux poisons, cités à $31,25 \%$ par les professionnels. Les collections paléontologiques et 
minéralogiques sont susceptibles de présenter des spécimens irradiés ou des minerais d'uranium brut. Les sels de radium, très utilisés à cause de leur luminescence spontanée, sont signalés dans les objets fluorescents des collections industrielles et techniques, et les instruments médicaux liés à la radiothérapie sont, peuvent, eux aussi, être irradiés.

Enfin, $25 \%$ des interrogés incluent l'amiante dans la liste des poisons. Imputrescible, incombustible et isolante, cette matière a largement été utilisée dans les collections industrielles. Le musée de Bièvres ainsi que les réserves du musée des Arts et Métiers possèdent tous les deux des objets amiantés, qu'il s'agisse, dans le cas du premier, d'appareils photographiques construits entre 1930 et 1970, ou, dans le cas du deuxième, de chrysotile (forme naturelle de l'amiante) pur, de rhéostats, ou d'objets du quotidien composés d'amiante, cependant plus stable car aggloméré.

11 Les poisons dans les collections sont une réalité historique, aujourd'hui mieux documentée. Cependant, les mentalités restent encore très figées, entre un déni menaçant la santé des professionnels et une prudence excessive affectant leurs conditions de travail et l'existence même des "collections poison".

\section{L'état des lieux de la prise de conscience des poisons}

\section{Les comportements à risques}

12 Au cours de leur carrière, les professionnels interrogés ont été témoin du manque de prise de conscience sérieuse de l'existence des poisons. La minimisation du problème ("on en meurt pas"), voire même le fatalisme ("c'est trop tard") auxquels ils ont été confrontés, couplés au manque de formation ou d'information, sont responsables des pratiques à risques constatées dans leurs environnements professionnels.

Parmi les situations rencontrées, les personnes interrogées constatent majoritairement un manque de protection personnelle de la part des professionnels de la conservationrestauration ou des sociétés de transport des œuvres, dans le cadre des manipulations réalisées sans protection des mains ou des voies respiratoires. De plus rares cas indiquent une exposition potentielle du public, sans protection, à des spécimens intoxiqués, dans le cadre de visites d'espaces de travail. Enfin, les comportements à risque s'observent aussi dans l'absence de délimitation des espaces définis, en raison d'une non-séparation des zones de travail et des zones de vie (utilisation d'un même évier pour nettoyer la vaisselle et le matériel de travail, ou de mêmes tables pour les repas et pour la restauration de spécimens).

14 Cette inconscience prend sa source dans le manque d'information autour de l'impact réel des poisons dans les collections. Problématique récente, elle n'a pas fait l'objet d'analyses scientifiques permettant de lier directement des maladies professionnelles à l'exposition à ces "collections poison", se limitant, dans la majorité des cas, à des attributions empiriques. Pourtant, l'impact des poisons est une réalité indiscutable, bien que mal connue : à Nantes, l'origine professionnelle d'un mésothéliome a été reconnue chez une ancienne employée des archives départementales de LoireAtlantique, après manipulation d'archives non-dépoussiérées ayant été conservées dans un bâtiment amianté ${ }^{3}$. Conscient de ces problématiques, le muséum d'Histoire naturelle de Rouen a lancé, dès 2010, une campagne d'analyse et de détection des 
produits chimiques sur ses propres collections, en partenariat avec l'INSA et le Département de Maîtrise des Risques Industriels et Environnementaux ${ }^{4}$.

Thermomètres et baromètres au mercure du musée des Arts et Métiers, de gauche à droite : Baromètre de démonstration ( $\mathrm{n}^{\circ} \mathrm{d}^{\prime}$ inventaire 08518-0000), Baromètre à double colonne (08761-0000), Baromètre portatif à siphon de Gay-Lussac (02628-0000), Baromètre de voyage avec themomètre de Mossy (01583-0000), Themomètre ou baromètre (brisé) (35336-0000), Grand thermomètre divisé sur cuivre dans son chassis (04252-0000).

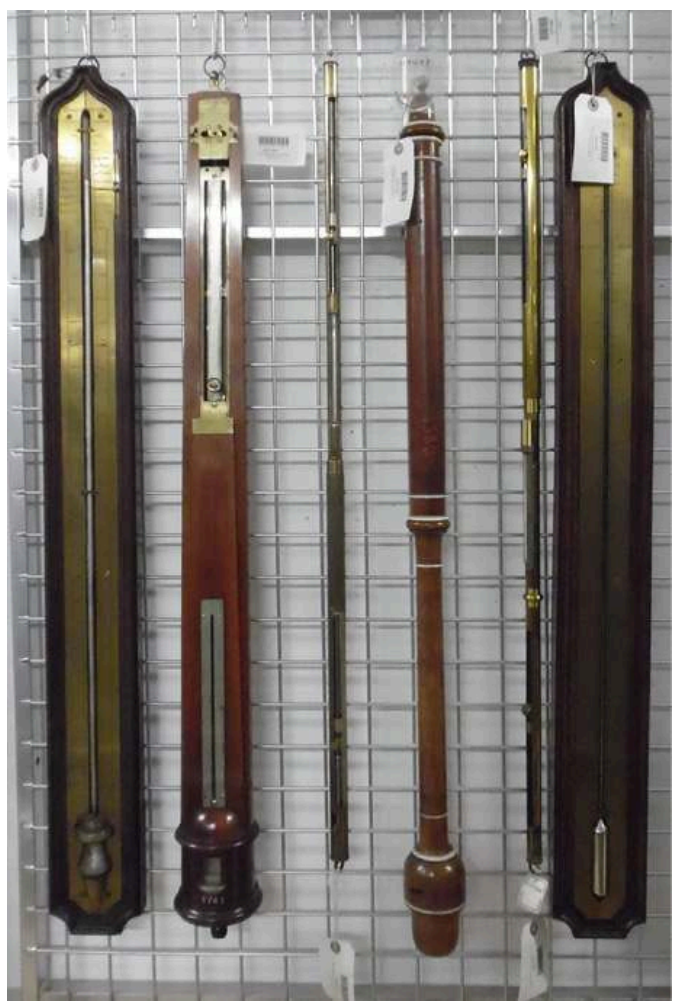

(c) Blandine Marcé

\section{L'excès de prudence}

15 Cette méconnaissance débouche également sur un excès de prudence qui menace gravement l'existence de ces collections. Les lourdes interdictions frappant la conservation ou l'utilisation de certains éléments toxiques, à l'image du décret $\mathrm{n}^{\circ}$ 96-1133 du 24 décembre 1996 relatif à l'interdiction de l'amiante, pousse les professionnels à se rassembler pour dénoncer les contradictions de telles mesures avec la conservation ou l'acquisition de certains patrimoines ${ }^{5}$. L'aspect fantasmé du "poison", trop souvent associé à de célèbres faits divers, pousse certains établissements à vouloir se débarrasser de leurs collections sur la simple suspicion de leur toxicité. La présentation générale du colloque des $6^{\text {es }}$ journées professionnelles de la conservationrestauration formule ainsi ces questionnements : "Comment les identifier ? Comment les traiter ? Faut-il conserver ces biens potentiellement nocifs ? [...] Faut-il s'acharner à trouver des méthodes de conservation ou se résoudre à abandonner à son sort tout un pan de la culture d'aujourd'hui ?". 
Boîte de câbles Fortin-Hermann présentant des pulvérulences de plomb ( ${ }^{\circ}$ d'inventaire 15815-0000).

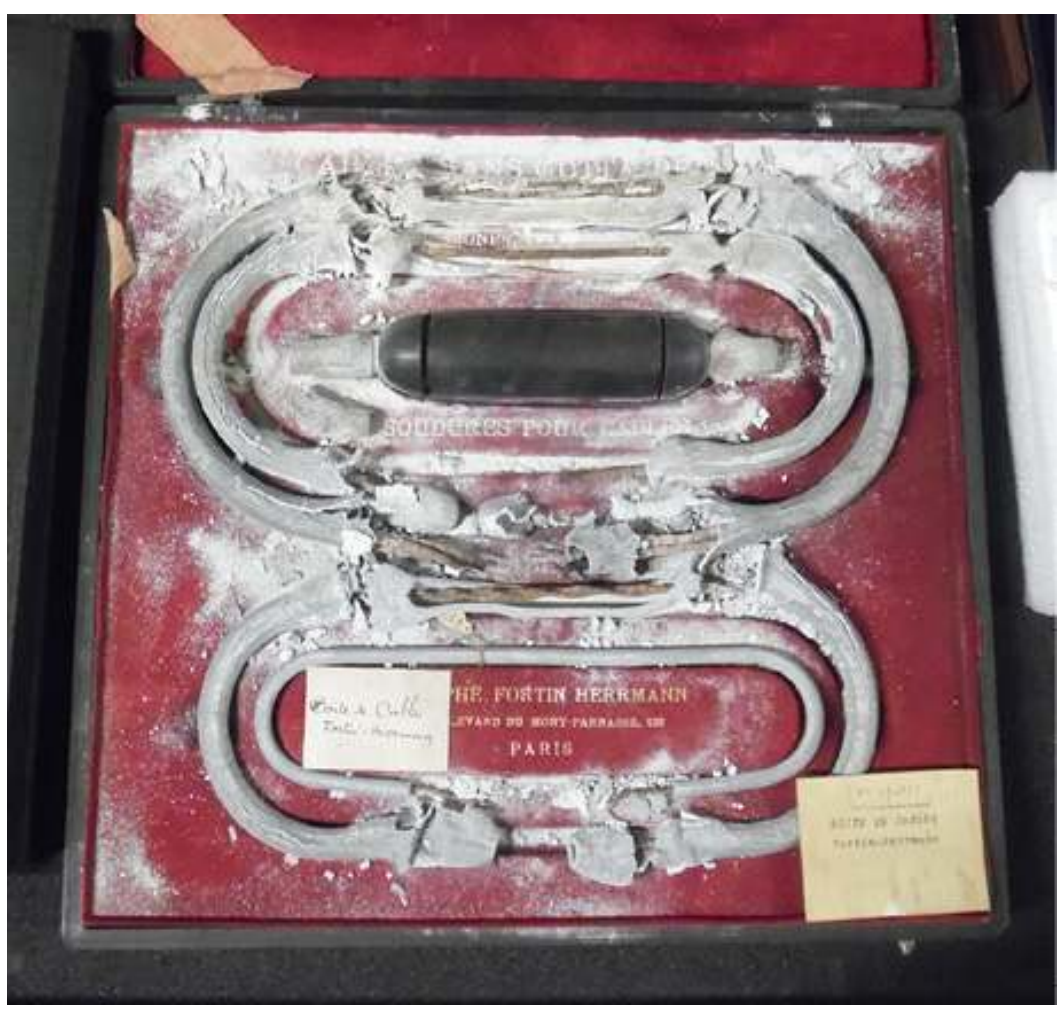

(c) Blandine Marcé

\section{Une question problématique}

La question des poisons est difficile, car elle demande une vision mesurée et globale, qui ne peut trouver de réponse dans le "tout ou rien".

La toxicité des collections n'est pas unilatérale : on ne peut placer, sur le même niveau de dangerosité, un spécimen récemment naturalisé ayant été dépoussiéré, et un spécimen $\mathrm{du} \mathrm{XIX}^{\mathrm{e}}$ siècle fortement encrassé. Leur détection même est difficile, en raison de l'inégale répartition des concentrations des poisons sur un seul et même spécimen ${ }^{6}$, qui peut facilement fausser les résultats. Bien qu'il existe actuellement des solutions de stockage permettant d'atténuer l'impact des collections les plus touchées (isolation des spécimens amiantés ou radioactifs dans des sacs Minigrip ou des pièces à part, balises...), la séparation pure et simple entre les spécimens contaminés et les spécimens sains, en plus d'être dangereuse car menant à croire que les spécimens "sains" sont complètement hors de danger, pose des problèmes de faisabilité, de coût et d'espace.

Face aux problématiques posées par les "collections poison", la solution semble résider davantage dans une plus grande information du personnel, dans la mise en place d'un repérage clair et dans l'emploi systématique d'équipements de protection adaptés. 


\section{Les mesures à mettre en place}

\section{Identifier facilement le risque et informer rapidement}

19 À court terme, la première étape est de repérer les collections grâce à l'histoire des biens culturels concernés. Savoir que l'arsenic, le formol et le chlorure mercurique ont été utilisés pour leurs propriétés insecticides et conservatives, et que l'amiante, le mercure liquide, le plomb et les objets radioactifs ont été employés pour leurs propriétés physiques permet de prendre conscience de leur présence à grande échelle. La documentation des collections, même de manière générale, est une nécessité préalable à toute mesure concrète, pour éviter les écueils qu'offre la méconnaissance des poisons.

Une fois que les collections concernées ont été identifiées, il s'agit de prendre des mesures préventives pour informer l'ensemble du personnel sur les potentiels dangers d'intoxication en facilitant le repérage des objets concernés. La méthode de signalisation est laissée à la discrétion des établissements, mais doit impérativement préciser les risques de manière évidente (ex : pictogrammes "toxique" ou "nocif"), tant sur des étiquettes que sur les registres d'inventaire, ou sur une base de données informatisée des collections, si elle existe. Le musée des Arts et Métiers a ainsi suggéré l'instauration d'un code couleur pour l'étiquetage des objets concernés, après réflexion dans le cadre du Plan de Prévention des Réserves. 
Exemple d'étiquette à code-barres mise en place dans le cadre du récolement décennal des collections aux réserves du musée des Arts et Métiers. Si un objet est identifié comme contenant une substance toxique, une nouvelle étiquette portant la mention du biocide (plomb, amiante, mercure) est imprimée, avec, à l'arrière, un sigle coloré reconnaissable. Sur la base de données informatisée des collections du musée, le toxique est indiqué en couleur rouge et la case "Constat d'État" remplie avec la mention "à surveiller".

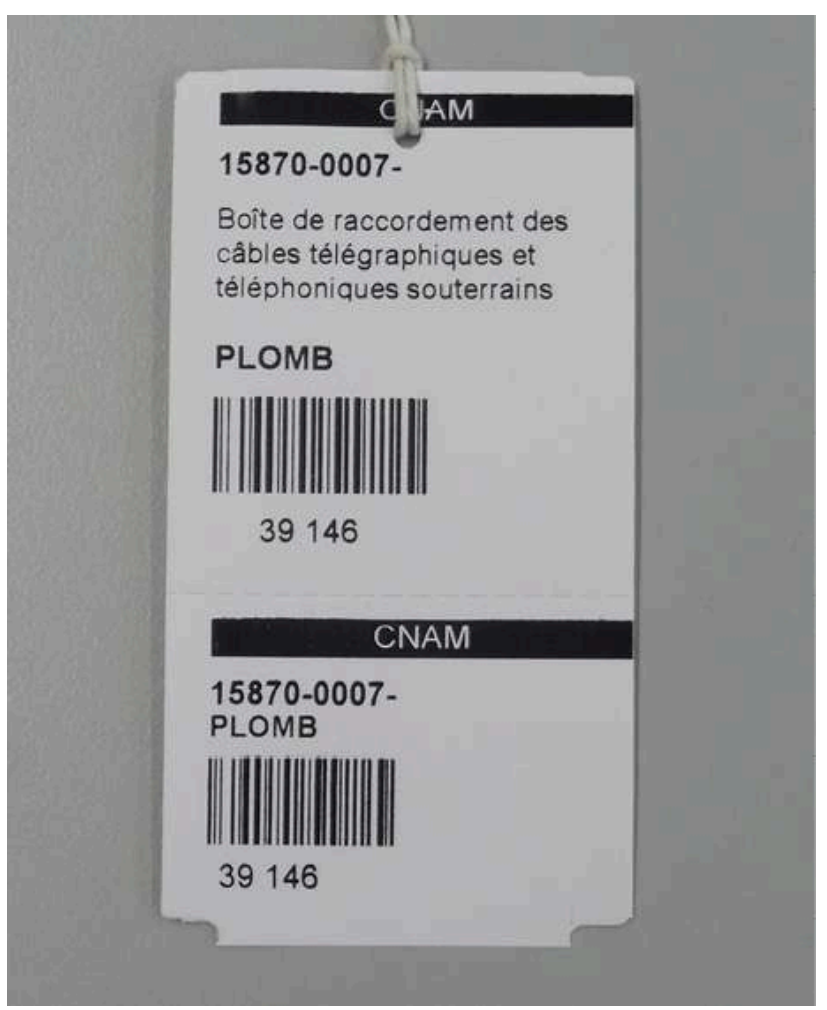

(c) Blandine Marcé

21 Un rappel physique des règles et comportements à suivre, à travers des fiches signalétiques disposées dans les espaces de travail, peut compléter efficacement le repérage physique des collections. Le Muséum national d'Histoire naturelle en fournit un bon exemple : à l'entrée du laboratoire de restauration des collections en fluide, un panneau informe, tout d'abord, de la présence de formol dans les locaux et souligne la nécessité de respecter les consignes de sécurité. Puis, à l'intérieur, une fiche résumant les modes de pénétration du formol (inhalation, cutané, oculaire), et les équipements adaptés en fonction de ces modes, est accrochée au mur, non loin de la fiche résumant les consignes opératoires élémentaires (allumer la hotte, comment ramasser le formol...), les consignes générales pour la manipulation (équipements obligatoires, gestes à proscrire), la manière d'éliminer les déchets et, enfin, un panneau indiquant qui contacter en cas de premiers secours, et pour quel type d'accident. Imprimées sur papier, ces fiches sont économiques et simples à mettre en place.

Enfin, une formation et information doit être faite en amont sur le comportement à adopter et les risques. Dans l'article L.230-2 du Code du Travail, il est précisé que : "Le chef d'établissement doit prendre les mesures nécessaires pour assurer la santé et la sécurité du personnel", aussi bien sur les points de la sensibilisation que de la mise en place des moyens de protection collective et de protection individuelle. De fait, la charge revient aux gestionnaires des collections, mais également aux différents acteurs de la sécurité et de la prévention, comme les comités d'hygiène et sécurité (CHSCT). 


\section{Protéger et limiter les accidents}

isque fort de contamination existe pour les personnes manipulant directement les œuvres, qu'il s'agisse des restaurateurs, conservateurs, chercheurs, régisseurs ou, le cas échéant, agents responsables du ménage. Un équipement de protection est donc absolument obligatoire pour limiter la pénétration des poisons dans l'organisme.

Les fiches signalétiques prévenant d'un danger toxique (ici, le formol) sont présentées dès l'entrée dans le laboratoire de restauration et préparation des collections en fluide du Muséum national d'Histoire naturelle de Paris.

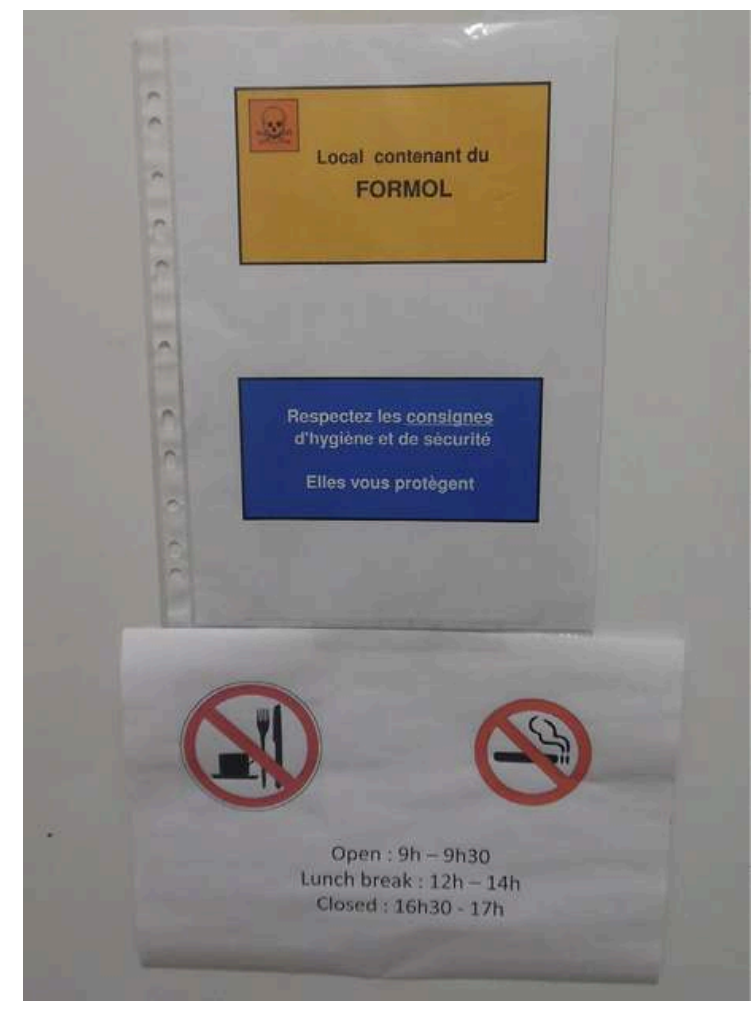

(C) Blandine Marcé

\section{Les équipements de protection individuelle}

Les protections doivent être utilisées en fonction du type de contamination (cutanée inhalation, ingestion) et de la dangerosité de la manipulation. De manière générale, le port des gants doit être obligatoire et systématique en cas de manipulation de spécimens intoxiqués, qu'il s'agisse d'une restauration ou d'un déplacement d'œuvres non-conditionnées. Les gants en nitrile sont préférables aux gants en latex ou en coton en raison de leur étanchéité. Le port d'une blouse lavable est recommandé pour éviter la dispersion des poussières intoxiquées. Les blouses de type Tyvek présentent un certain nombre d'avantages, par leur résistance à l'eau, aux produits chimiques, et leur légèreté. Pour la manipulation de l'amiante, il est recommandé de porter une combinaison intégrale jetable.

Pour la protection des voies respiratoires, il est recommandé de porter un masque pour la manipulation d'objets empoussiérés (poussières d'arsenic, d'amiante), de type FFP3 
(pour l'amiante et les particules fines) ou FFP2. Dans le cas des collections en fluide, des masques anti-gaz ou ayant des cartouches à solvant sont obligatoires. Ils doivent être correctement ajustés, ne servir qu'à la protection d'un seul type de toxique, et être régulièrement changés (cartouches) ou lavés (masques non-jetables). Pour des manipulations plus légères, des demi-masques en coton jetable ou en pâte compressée peuvent également être utilisés.

Pour certaines opérations, comme le dépoussiérage des collections, mais surtout dans les cas de dégagement de vapeurs toxiques (collections en fluide ouvertes, mercure liquide, fluides manipulés...), il est conseillé de porter des lunettes de protection à coques latérales pour prévenir les projections de liquide, ou des lunettes spécifiques contre les gaz. Les restaurateurs de collections en fluide doivent idéalement porter des bottes ou sur-chaussures de protection en cas de fuite du liquide ou d'accident.

Laboratoire de préparation et de restauration des collections en fluide du Muséum national d'Histoire naturelle de Paris. Le muséum possède un système perfectionné pour protéger les professionnels : en haut à droite, un système de ventilation adapté pour la manipulation du formol, au dessus d'une sorbonne. Au dessus de la table de dissection et préparation, un boa (ou système d'aspiration souple). Au mur, affichage de consignes de sécurité à côté des espaces de travail.

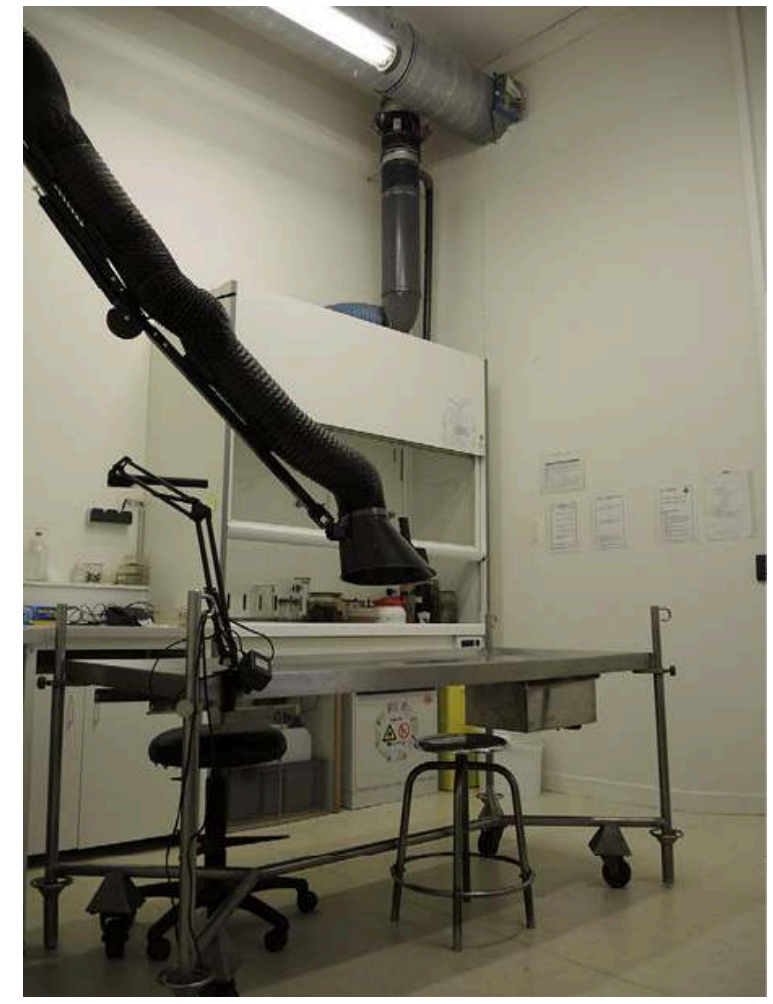

(c) Marc Herbin

\section{Les gestes à éviter}

Des gestes simples sont à rappeler pour éviter une contamination accidentelle. Ne pas porter les mains à sa bouche ou à son visage (se ronger les ongles, placer une mèche de cheveux derrière son oreille, mordiller un crayon...), ne pas fumer, boire ou manger dans les locaux de manipulation des toxiques, penser à enlever ses gants quand on manipule d'autres objets que le spécimen ou les instruments en lien avec sa 
restauration (poignée de porte, portable, appareil photo...) sont autant de précautions qui limitent l'impact des collections contaminées sur la santé.

\section{La séparation des espaces collectifs} Les éléments jetables utilisés pour nettoyer ces espaces (lingettes, sacs d'aspirateurs...), doivent être jetés dans des poubelles dédiées aux produits toxiques, dont l'élimination doit idéalement être réalisée par une entreprise spécialisée.

\section{Méthodes de détection chimique et base de données partagée}

31 À moyen et long terme, des solutions pour détecter les biens culturels à risques et accroître la sensibilisation et l'information sur la question des poisons sont envisageables.

Le repérage des manifestations physiques de la présence des poisons dans les collections (tâches gris-noir sur les herbiers indiquant la présence de mercure, cristallisation blanche de l'arsenic sur les espèces contaminées, pulvérulence du plomb corrodé...) peut être complété grâce à des méthodes de détection instrumentales et chimiques. Les spots-tests, ou papiers tournesols, changeant de couleur en présence d'un certain type de toxique (arsenic, mercure, plomb, argent...) à l'aide d'un réactif, sont une solution économique très souvent utilisée par les musées. Pour les fluides, le réactif de Schiff, devenant rose au contact du formaldéhyde, peut être utilisé sur des échantillons donnés.

Faciles d'emploi, le compteur Geiger-Müller et les radiamètres permettent de détecter facilement les rayonnements alpha, bêta et gamma en évaluation directe, sans échantillon.

Rapide et non-destructive, mais plus coûteuse, la spectrométrie de fluorescence X peut représenter un investissement financier valable sur le long terme pour le repérage des métaux lourds et métalloïdes comme l'arsenic, le mercure, ou le plomb. Pouvant être portable, et permettant une intervention directe sur les collections et une réponse rapide ( 1 minute par test contre jusqu'à 30 minutes pour les spots tests), elle nécessite cependant une certaine formation pour interpréter ses résultats. 
Les réserves de pièces anatomiques en fluide du Muséum national d'Histoire naturelle de Paris sont équipées de lampes anti-déflagrantes pour limiter les risques d'explosion liés aux vapeurs toxiques.

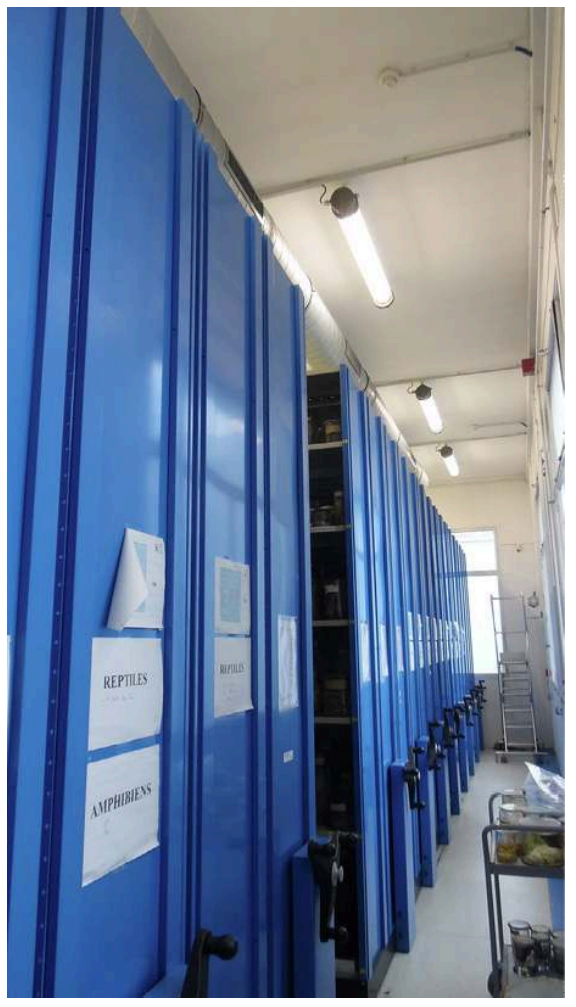

(C) Blandine Marcé

Ces formations manquent actuellement en France. En effet, si l'INP présente ponctuellement des formations payantes destinées aux professionnels sur les questions des collections contaminées (fluides en 2008, spécimens naturalisés en 2011 et 2012, amiante en 2015), il n'existe pas de formation systématique en amont ayant pour objet la toxicité des collections. À l'avenir, il serait pertinent d'envisager des interventions plus générales et accessibles, à des niveaux moins spécialisés, pour rappeler l'existence des "collections poison" et fournir une documentation aux futurs professionnels.

La documentation sur les poisons dans les musées est aujourd'hui encore très spécialisée, dispersée et difficilement accessible. Les fiches toxicologiques de l'INRS, bien qu'utilisées comme référent principal par les professionnels, n'apportent qu'un éclairage scientifique et chimique du poison sous sa forme pure, qui pêche par son application concrète.

La solution serait de créer une base de données en ligne, ou un site Internet, exclusivement dédié aux "collections poison", permettant de regrouper des fiches d'information par mots-clés. À la différence des fiches de l'INRS, la recherche pourrait se faire soit par le poison (Mot clé "Mercure", "Amiante", "Formol"...), soit par le type de collections ("Taxidermie", "Bocaux", "Fluides", "Industrie"), soit par l'objet luimême ("Animal naturalisé", "Herbier", "Moteur de voiture"...), et permettrait d'accéder directement à une fiche claire et rapide de description du poison, permettant de connaître le type d'objets concerné, les risques encourus, et les mesures de protection à prendre. 
38 Nous proposons en exemple deux types de fiches en ligne. La première, très synthétique, cite le type d'objets concerné, l'apparence que peut prendre le poison quand il est visible et sa méthode de contamination, et, enfin, les équipements personnels recommandés en fonction du type de manipulation (prolongée ou non). La deuxième fiche, plus détaillée, concerne divers aspects des collections. Elle reprend, dans une première partie, la typologie de l'objet et son apparence sous sa forme toxique (poussières, dégradations, fuite...), puis traite de sa toxicité en rappelant ses effets à court et à long terme, ses valeurs limites d'exposition, et ses méthodes de contamination, avant de terminer par une liste de solutions pour protéger le personnel, tant pour la protection personnelle que la protection collective, la détection et le stockage.

Le site, idéalement participatif, pourrait enfin être complété par une base iconographique, une bibliographie thématique regroupant les différents travaux déjà réalisés sur la question des poisons dans les musées (mémoires, articles, ouvrages...), ainsi qu'un agenda des rencontres organisées autour de la question.

Exemples de fiches synthétique et détaillée concernant la radioactivité

\begin{tabular}{|c|}
\hline RADIOACTIVITÉ \\
\hline Type d'objets \\
\hline $\begin{array}{l}\text { Minéraux radioactifs, fossiles, sels de radium présents dans des produits } \\
\text { luminescents (aiguilles de montre, panneaux de signalisation, etc), } \\
\text { collections médicales (radiothérapie, curiethérapie), appareils électriques } \\
\text { (paratonnerres, détecteurs incendie), appareils photographiques, } \\
\text { fontaines à radium, verre d'urane ou ouraline. }\end{array}$ \\
\hline Apparence \\
\hline $\begin{array}{l}\text { Pas d'apparence particulière. Liée à la collection, noms à consonance } \\
\text { radioactive (radium, uranium, etc). Parfois, une luminescence prolongée. } \\
\text { Couleur vert-jaune de l'ouraline ou de l'urane. }\end{array}$ \\
\hline Forme toxique \\
\hline $\begin{array}{l}\text { Poussières radioactives (minéraux ou poussière d'usure contaminée), } \\
\text { rayonnement radioactif. }\end{array}$ \\
\hline Protection personnelle \\
\hline $\begin{array}{l}\text { Pour le déplacement d'objets: gants jetables en nitrile, blouse ou } \\
\text { combinaison lavable. Masque dans le cas de collections empoussiérées. }\end{array}$ \\
\hline $\begin{array}{l}\text { Pour la restauration ou le chantier des collections (manipulation } \\
\text { prolongée) : gants en nitrile, blouse ou combinaison lavable, masque dans } \\
\text { le cas de collections très empoussiérées, protection des cheveux dans le } \\
\text { cas d'un dépoussiérage des collections. }\end{array}$ \\
\hline
\end{tabular}




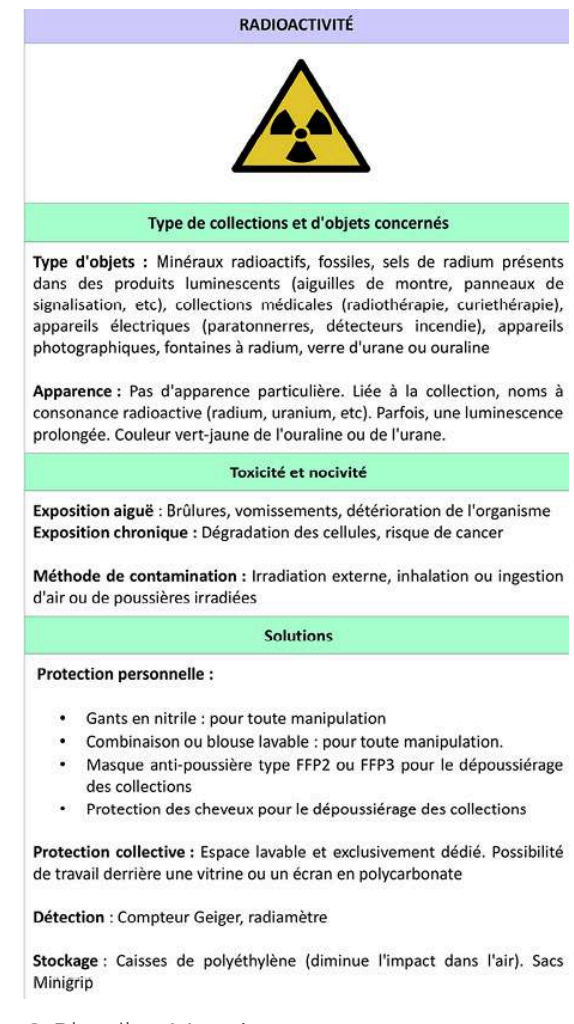

(c) Blandine Marcé

\section{Conclusion}

Difficile à résumer en raison de la variété de ses manifestations et des situations concernées, nous pouvons tirer de cet état des lieux sur la problématique des poisons et des témoignages recueillis une idée principale : la communication sur les "collections poison" doit être faite à tous les niveaux. À court terme, des solutions simples d'identification et d'information des acteurs du patrimoine (repérage des collections, fiches signalétiques, protection systématique) peuvent permettre de diminuer sérieusement les risques que courent actuellement les professionnels, dans l'attente de campagnes de détection plus vastes, de solutions de stockage adaptées, et de la mise en place d'une documentation commune sur la question.

41 Suivant l'exemple donné par les musées comme Bièvres ou le muséum d'Histoire naturelle de Rouen, il est à espérer, qu'à l'avenir, les comportements à risque ne soient plus constatés, du fait d'une information systématique, efficace, accessible et répandue. La diffusion de la problématique à large échelle permettrait d'approfondir et d'élargir le sujet des poisons dans les musées, touchant des domaines extrêmement divers. En effet, si ce mémoire a choisi de traiter les poisons constitutifs des collections, en raison de leur aspect doublement problématique (patrimonial et sanitaire), restent encore à traiter les poisons utilisés lors des traitements des collections (créosote de hêtre, DDT, lindane...), la rémanence de ces poisons dans l'environnement et la contamination des espaces étant tout aussi néfaste. 


\section{BIBLIOGRAPHIE}

Aubagnac, G. Les risques associés à la conservation des collections militaires et techniques, $L a$ Lettre de l'Ocim, n¹68, 2016, pp. 16-20.

Dangeon, M. Contamination des collections naturalisées traitées aux biocides et mesures de conservation préventive, CeROArt, mis en ligne le 25 février 2016, journal.openeditions.org/ ceroart.

Daval, M. Les substances radioactives dans les objets patrimoniaux, CeROArt, mis en ligne le 2 avril 2014, journal.openeditions.org/ceroart.

Defendini, L. L'impact des collections patrimoniales sur la santé, La Lettre de l'Ocim, n¹68, 2016, pp. 12-15.

Échinard, J. L'amiante dans les collections patrimoniales : l'exemple du musée français de la Photographie de Bièvres, in Dussère, F. (dir.) La conservation-restauration et la sécurité des personnes, actes de colloque, 2010, $105 \mathrm{p}$.

Herbin, M. La conservation des collections en fluide, CeROArt, mis en ligne le 18 août 2013.

Illes, V. Guide de manipulation des collections. Somology, 2004, 128 p.

Marcé, B. Les "collections poison" : typologie, enjeux de conservation et gestion des risques, Mémoire de l'École du Louvre, 2016, 60 p.

Marcotte, S., Estel, L., Leboucher, S. et Minchin, S. Évaluer la pollution de l'air au muséum d'Histoire naturelle de Rouen, La Lettre de l'Ocim, nº145, 2013, p. 13.

Péquignot, A. Évaluation de la toxicité des spécimens naturalisés, La Lettre de l'Ocim, n¹16, 2008, pp. 4-9.

Pfister, A.-L. L'influence des biocides sur la conservation des "naturalia", Mémoire de fin d'études, Haute École d'Arts appliqués Arc, Neuchâtel, 2008, 188 p.

Tarchini, A. Le mercure dans les collections du patrimoine technique et industriel : problématiques de conservation, Mémoire de fin d'études, filière Conservation-Restauration, orientation Objets scientifiques, techniques et horlogers, Haute École d'Arts appliqués Arc, Neuchâtel, 2006.

Fiches toxicologiques, INRS : www.inrs.fr/publications/bdd/fichetox.html

Ministère de la Culture et de la Communication, dossier et vidéos du colloque des $6^{\text {es }}$ journées de la conservation-restauration :

www.culturecommunication.gouv.fr/Thematiques/Conservation-restauration/Journeesprofessionnelles/conserver-malgre-tout-Limites-

et-defis-videos-des-journees-professionnelles-2016

\section{NOTES}

1. De Beaulieu, M. Étude et restauration d'un spécimen naturalisé et d'un squelette de lémuriens du Muséum national d'Histoire naturelle. Étude et mise en place d'un dispositif de protection contre l'arsenic à l'usage des restaurateurs, Mémoire de fin d'études, INP, septembre 2010, 269 p.

2. www.inrap.fr/la-sepulture-de-louise-de-quengo-dame-du-xviie-siecle-11010 
3. Fascicule des $6^{\mathrm{es}}$ journées professionnelles de la conservation-restauration, article "Amiante et patrimoine culturelle", p. 42.

4. Minchin, S. De l'analyse à la prévention, La Lettre de l'Ocim, $\mathrm{n}^{\circ} 168$, [En ligne], http:// journals.openedition.org/ocim/1706.

5. Fascicule des $6^{\text {es }}$ journées professionnelles de la conservation-restauration, article "Amiante : l'archiviste et l'invisible", p. 16.

6. De Beaulieu, p. 49.

\section{RÉSUMÉS}

À partir de témoignages de professionnels des musées, l'auteur dresse un tableau des dangers liés à la manipulation et à l'exposition de produits toxiques présents dans les collections. Face à ce qui demeure encore parfois aujourd'hui des comportements à risques, cette étude propose une liste de bonnes pratiques préconisant des solutions pour l'identification de ces "poisons" et l'information des personnels. Elle plaide pour la mise en place d'une véritable politique de prévention.

\section{INDEX}

Mots-clés : Collections, santé, conservation

\section{AUTEUR \\ BLANDINE MARCÉ}

Assistante administratrice de la base de données Archange au ministère des Armées. marce.blandine@gmail.com 